\title{
Propagation Effects in Superfluorescence
}

\author{
R. Michalska-Trautman \\ Institute of Physics, Polish Academy of Sciences, Aleja Lotnikow 32/46, PL-02668, Warsaw, Poland \\ (Received September 6, 2016) \\ The evolution of a superfluorescent pulse is studied in the region of large sample lengths. A solution to the \\ Maxwell-Bloch semiclassical equations is found by using the inverse scattering method. It has the form of a one- \\ soliton solution with varying parameters. Dependence on the pulse width and the retarded time is found without \\ further approximations. These parameters are shown to be independent of the inhomogeneous broadening time. \\ Their values are in good agreement with experiment. Asymmetry of the pulse is also investigated.
}

DOI: 10.12693/APhysPolA.130.734

PACS/topics: 42.65.Sf, 42.65.Tg

\section{Introduction}

At the second stage of superfluorescence (SF), when small, but macroscopic, polarization is already formed in the medium, the evolution of the electromagnetic (EM) field is governed by the semiclassical MaxwellBloch (MB) equations. These equations can be integrated by the inverse scattering method (ISM).

In a previous paper [1] exact conservation laws for the SF process have been derived. It was shown there that the SF radiation starts at a certain minimal length of propagation. In the present paper, the evolution of the pulse shape is studied for lengths larger than the threshold value. The method of solving the evolution equation is based on ISM. It is close to that used by Steudel [2], but differs from it in the way approximations are made. A priori assumptions about orders of magnitude are avoided.

The EM pulse is found in the form of a one-soliton solution with varying parameters. The retarded time and pulse width are calculated easily. Asymmetry of the pulse in time is discussed. To a good approximation, the retarded time and pulse width are shown to be independent of the inhomogeneous broadening.

In Sect. 2, earlier results on MB equations and ISM are briefly reviewed. In Sect. 3, the form of the EM pulse is found by the use of stationary phase method applied to the integral equations of ISM. The only assumption, used to establish this solution, is the asymptotic region of large $x$.

Variation of soliton parameters is studied in Sect. 4. Simple dependence of the retarded time and pulse width on $x$ and characteristic parameters are established. Finally, in Sect. 5, the results are compared with other theoretical results and experimental data.

\section{The Maxwell-Bloch equations}

Interaction of the electromagnetic field with two-level atoms in the slowly varying envelope approximation is described by the Maxwell-Bloch equations which, in dimensional notation, are of the form

$$
\begin{aligned}
& E_{x}=\langle\lambda\rangle, \\
& \lambda_{t}=-2 \mathrm{i} \omega \lambda+N E,
\end{aligned}
$$

$$
N_{t}=-\frac{1}{2}\left(E \lambda^{*}+E^{*} \lambda\right) .
$$

Here $E(t, x), \lambda(t, x, \omega)$ and $N(t, x, \omega)$ denote, respectively, the field envelope, complex polarization, and population inversion in the medium; star denotes complex conjugation and $t$ is the retarded time. Angular brackets denote averages over the inhomogeneously broadened atomic line,

$$
\langle\lambda\rangle=\int_{-\infty}^{\infty} \lambda(\omega) g(\omega) \mathrm{d} \omega,
$$

with the Gaussian distribution

$$
g(\omega)=\frac{T}{\sqrt{\pi}} \mathrm{e}^{-\omega^{2} T^{2}},
$$

where $2 \omega$ is the difference between the atomic frequency and the central frequency $\omega_{0}$ of the transition. All quantities are dimensionless, scaled by the so-called coherence time

$$
\tau_{\mathrm{c}}=\left(2 \pi n \mathcal{P}^{2} \omega_{0} / \hbar\right)^{-1 / 2}
$$

or by $c \tau_{0}$. Here $\mathcal{P}$ denotes the transition matrix element, $n$ is the density of atoms and $c$ is the velocity of light.

The initial boundary conditions for SF read

$$
\begin{aligned}
& E(t, 0)=E(0, x)=0, \\
& \lambda(0, x, \omega)=\lambda_{0}, \quad N(0, x, \omega)=N_{0} .
\end{aligned}
$$

In general, $\lambda_{0}$ depends on $x$ and $\omega$ and is a random function.

The system (1)-(3) is integrable by the inverse scattering method, see, e.g. [3]. The evolution in $x$ of the scattering data for SF is known thanks to the works of Gabitov et al. [4] and Steudel [2]. In particular, for $\lambda_{0}$ uniform in $x$ and $\omega$, the exact solution for the scattering amplitude reads [2]:

$$
r(\zeta, x)=\sqrt{\gamma}\left(\mathrm{e}^{W x}-1\right) /\left(1+\gamma \mathrm{e}^{W x}\right),
$$

where $\gamma=\tan ^{2} \frac{1}{2} \Theta, \Theta=\sin ^{-1} \lambda_{0}, W=\frac{1}{2} \sqrt{\pi} T w(\zeta T)$ and $w$ denotes the complex probability error function [5]:

$$
w(z)=\frac{\mathrm{i}}{\pi} \int_{C} \frac{\mathrm{e}^{-t^{2}}}{z-t} \mathrm{~d} t .
$$

Discrete eigenvalues $\zeta_{j}$ are given by the condition

$$
x w\left(\zeta_{j} T\right)=\delta+\mathrm{i}(2 j+1) \pi, \quad j=0,1, \ldots,
$$
where $\delta=\frac{1}{2} \ln \gamma^{-1}$. 
Conservation laws for SF were derived in [1] with the use of (9). From the exact expression for the energy transmitted to the medium (cf. Eq. (3.29) in [1]), it follows that maximum of radiation, corresponding to the Dicke half-excited state, occurs at

$$
x_{m}=\frac{1}{\sqrt{\pi} T} \ln \gamma^{-1} .
$$

It may be interpreted as the threshold length for SF. It seems natural, therefore, to look for the pulse solution in the region $x>x_{m}$.

\section{The inverse problem}

To reconstruct the field from the scattering data by the ISM one has to solve a system of integral equations that are of the form given in $[6,7]$,

$\chi_{2}(\zeta)=-\frac{1}{2 \pi \mathrm{i}} \int_{C} \frac{1}{z-\zeta T} r(z) \chi_{1}(z / T) \mathrm{e}^{-2 \pi \mathrm{i} z t / T} \mathrm{~d} z$,

$\chi_{1}(\zeta)=1-\frac{1}{2 \pi \mathrm{i}} \int_{\bar{C}} \frac{1}{z-\zeta T} \bar{r}(z) \chi_{2}(z / T) \mathrm{e}^{2 \pi \mathrm{i} z t / T} \mathrm{~d} z$,

where $\chi_{1}$ and $\chi_{2}$ are connected to the Jost function $\chi^{+}$ defined in [2]; see also eq. (2.13) in [1]. One has

$$
\chi_{1}=-\chi_{22}^{+} \mathrm{e}^{\mathrm{i} \zeta x}, \quad \chi_{2}=\chi_{21}^{+} \mathrm{e}^{-\mathrm{i} \zeta x} .
$$

The contour of integration $C$ (respectively $\bar{C}$ ) starts from $\zeta=-\infty+\mathrm{i} \epsilon$ (respectively $\zeta=-\infty-\mathrm{i} \epsilon$ ), passes above (respectively below) all poles of $r$ (respectively $\bar{r}$ ) and ends at $\zeta=+\infty+\mathrm{i} \epsilon$ (respectively $\zeta=+\infty-\mathrm{i} \epsilon$ ). The EM field $E$ is determined by the asymptotic form of $\chi_{2}$ for large $\zeta$,

$$
\chi_{2}=\frac{\mathrm{i}}{4 \zeta} E+\mathrm{O}\left(\zeta^{-2}\right) \text {. }
$$

The functions $\chi_{1}$ and $\chi_{2}$ are analytic in the upper and the lower halves of the $\zeta$-plane, respectively. Due to the exponential growth of $r$ for large $\bar{x}=\frac{1}{2} x T$ one can apply the stationary phase method [8,9] to the integrals (13) and (14). The stationary phase condition reads

$$
\sqrt{\pi} w^{\prime}-2 \mathrm{i} t / \bar{x} T=0 \text {. }
$$

Solving this equation one finds $z_{s}=\mathrm{i} \eta T$ and $\left.w^{\prime \prime}\right|_{z=z_{s}}<0$. If $z_{s}$ is above all the poles of $r$, then the contour of integration may be deformed to pass through the stationary point. This condition for $z_{s}$ is discussed in Sect. 5. The integral in Eq. (13) may be approximated by its leading term. Similarly, the path of integration in Eq. (14) can be deformed in the lower half-plane, where $z_{s}=-\mathrm{i} \eta T$. As a result, one gets, for large $\bar{x}$, the relations

$$
\begin{aligned}
& \chi_{1}=1-\mathrm{iRe}^{2 \eta t}(\mathrm{i} \eta T+\zeta T)^{-1} \chi_{2}(-\mathrm{i} \eta), \\
& \chi_{2}=\mathrm{iRe}^{2 \eta t}(\mathrm{i} \eta T-\zeta T)^{-1} \chi_{1}(\mathrm{i} \eta),
\end{aligned}
$$

where

$$
\begin{aligned}
& R=\frac{\mathrm{e}^{\sqrt{\pi} \bar{x} w_{s}}}{\sqrt{2 \pi \gamma^{-1} \bar{x}\left|w_{s}^{\prime \prime}\right|}\left(1+\gamma \mathrm{e}^{\sqrt{\pi} \bar{x} w_{s}}\right)}, \quad w_{s}=w\left(\mathrm{i} \eta_{s} T\right), \\
& w_{s}^{\prime \prime}=\mathrm{d}^{2} w /\left.\mathrm{d} z^{2}\right|_{z=z_{s}} .
\end{aligned}
$$

Solving Eqs. (17) and (18) for $\chi_{2}(-\mathrm{i} \eta)$ and $\chi_{1}(\mathrm{i} \eta)$ and using (15), one finds

$$
E=2 \eta \operatorname{sech} 2 \eta\left(t-\tau_{0}\right),
$$

where $\tau_{0}$ is defined by:

$$
\left[\ln (R / 2 \eta T)+2 \eta \tau_{0}\right]_{t=\tau_{0}}=0 .
$$

The solution (20) has the form of a one-soliton wave with varying parameters $\eta(x, t)$ and $\tau_{0}(x)$. These variations are studied in the next section. At this stage, I would like to emphasize that the only assumption used to find (20) has been that $\bar{x} \gg 1$. A similar result has been obtained by Steudel [2] under more stringent assumptions.

\section{Retarded time and pulse duration}

Considering Eqs. (16) and (21) at $t=\tau_{0}$ and defining:

$$
y=\left.\eta\right|_{t=\tau_{0}} T, \quad \tau=\tau_{0} / T,
$$

one obtains

$$
\begin{aligned}
& \sqrt{\pi} y w(\mathrm{i} y)=1-\tau / \bar{x}, \\
& 2 y \tau=\ln 2 y-\left.R\right|_{t=\tau_{0}},
\end{aligned}
$$

where $R$ is given by Eq. (19) and

$$
\left.w^{\prime \prime}\right|_{t=\tau_{0}}=\frac{4 y}{\sqrt{\pi}}\left[\sqrt{\pi} w(\mathrm{i} y)\left(\frac{1}{2} y^{-1}+y\right)-1\right] .
$$

By eliminating $\tau$ from Eq. (23) one gets the following equation for $y$ :

$$
F_{1}(y)=\frac{1}{4 \bar{x}}\left[\Gamma+\ln \bar{x}+F_{2}(y)\right],
$$

where

$$
\begin{aligned}
& F_{1}(y)=\sqrt{\pi} w(\mathrm{i} y)\left(\frac{1}{2}-y^{2}\right)+y, \\
& F_{2}(y)=\ln \left[y^{3}\left(\sqrt{\pi} w(\mathrm{i} y)\left(\frac{1}{2} y^{-1}+y\right)-1\right)\right],
\end{aligned}
$$

and $\Gamma=\ln \left(32 \sqrt{\pi} \gamma^{-1}\right)$. A computer plot of $y$ shows that this is a nearly linear function of $x$. The rate of growth slightly diminishes with growing $\bar{x}$. For large $y$ Eq. (26) can be given a simple form. The asymptotic behavior of the probability function is given by

$$
\sqrt{\pi} w(\mathrm{i} y)=y^{-1}\left(1-\frac{1}{2} y^{-2}+\frac{3}{4} y^{-4}+\ldots\right) .
$$

Therefore,

$$
F_{1}(y) \simeq y^{-1}, \quad F_{2}(y) \simeq \ln 2 y,
$$

and Eq. (26) reduces to

$$
y=\frac{4 \bar{x}}{\Gamma^{\prime}+\ln x-\ln y},
$$

where $\Gamma^{\prime}=\ln \left(16 \pi \gamma^{-1}\right)$. It follows that $Y=\bar{x} / y$ is a function of $\Gamma^{\prime}$ only, defined by the equation:

$$
Y-\frac{1}{4} \ln Y=\frac{1}{4} \Gamma^{\prime} .
$$

As a consequence, the duration of the pulse is independent of $T$ and varies linearly with $\bar{x}^{-1}$, while

$$
\eta \simeq x / 4 \Gamma^{\prime \prime}
$$

where $\Gamma^{\prime \prime}$ is defined by Eq. (31). Introducing (32) into Eq. (23) we find, with the same accuracy,

$$
\tau_{0}=\frac{1}{2} \Gamma^{\prime \prime 2} / x
$$

to be independent of $T$.

\section{Discussion}

The range of the parameter $\bar{x}$ for which the saddle point approximation can be applied is from $\bar{x}=\bar{x}_{m}$ at 
the threshold to $\bar{x}=\bar{x}_{s}$, where $\tau(x)$ saturates. Denoting by $\eta_{0}$ the imaginary part of the first discrete eigenvalue, we see that in this region $\eta / \eta_{0}>1$ and the discrete eigenvalues do not contribute to the solution (20).

The value of the constant $\Gamma^{\prime \prime}$ in the formula for the retarded time (23) is somewhat smaller than the one calculated by Steudel [2]. For the quotient $\tau_{p} / \sqrt{\tau_{D} \tau_{R}}$ I get a value 2.2 times larger than Steudel's. Differences between our results appear also in the shape of the pulse. I get a more marked asymmetry, of the order of $\left(\tau_{p} / \tau_{D}\right)^{2}$. In the approximation used in Sect. 4 , the values of $\tau_{D}$ and $\tau_{p}$ are independent of inhomogeneous broadening.

Using, in my formula, the experimental values of the parameters in the cesium vapor experiment referred to by Vrehen and Gibbs [10], I obtain

$$
\bar{x}_{m}=7, \quad \bar{x}_{s}=20, \quad \tau_{D}=11.9 \mathrm{~ns}, \quad \tau_{p}=3 \mathrm{~ns} .
$$

These numbers are larger than the measured values $\tau_{D}=$ $10 \mathrm{~ns}, \tau_{p}=2 \mathrm{~ns}$.

\section{Acknowledgments}

The author thanks Dr. H. Steudel for hospitality and stimulating discussions during her visit in Berlin. This research was supported in part by the Polish Committee for Scientific Research $(\mathrm{KBN})$ under grant No. 2.0480.91.01.

\section{References}

[1] R. Michalska-Trautman, Phys. Rev. A 46, 7270 (1992).

[2] H. Steudel, Quantum Opt. 2, 387 (1990).

[3] M.J. Ablowitz, H. Segur, Solitons and the Inverse Scattering Transform, Society for Industrial and Applied Mathematics, Philadelphia 1981.

[4] I.P. Gabitov, V.E. Zakharov, A.V. Mikhailov, Theor. Math. Phys. (USSR) 63, 328 (1985) [English translation].

[5] V.E. Zakharov, A.B. Shabat, Sov. Phys. JETP 34, 62 (1972).

[6] M.J. Ablowitz, D.J. Kaup, A.C. Newell, H. Segur, Stud. Appl. Math. 53, 249 (1974).

[7] Handbook of Mathematical Functions, Eds. M. Abramowitz, I.A. Stegun, Dover, New York 1970.

[8] N. Bleistein, R. Handelsman, Asymptotic Expansions of Integrals, Dover, New York 1975.

[9] L. Hörmander, Linear Partial Differential Operators, Vol. 1, Springer-Verlag, Berlin 1976.

[10] Q.H.F. Vrehen, H.M. Gibbs, J. Opt. Soc. Am. 68, 699 (1978). 